\title{
Renal function impairment predicts mortality in patients with chronic heart failure treated with resynchronization therapy
}

\author{
Edoardo Gronda ${ }^{1}$, Stefano Genovese ${ }^{1}$, Luigi Padeletti ${ }^{2,3}$, Francesco Cacciatore ${ }^{4}$, \\ Dino Franco Vitale ${ }^{4}$, Renato Bragato ${ }^{5}$, Lisa Innocenti ${ }^{2}$, Concetta Schiano ${ }^{6}$, \\ Linda Sommese ${ }^{7}$, Maria Rosaria De Pascale ${ }^{7}$, Luca Genovese ${ }^{1}$, \\ Pasquale Abete ${ }^{8}$, Francesco Donatelli ${ }^{1,9}$, Claudio Napoli ${ }^{6,7}$ \\ ${ }^{1}$ Department of Cardiovascular and Metabolic Disease, IRCCS MultiMedica, Sesto S. Giovanni, Milan, Italy \\ ${ }^{2}$ Chair and Division of Cardiology, University of Florence, Florence, Italy \\ ${ }^{3}$ Cliniche Gavazzeni, Bergamo, Italy \\ ${ }^{4}$ Fondazione Salvatore Maugeri-IRCCS, Telese, Benevento, Italy \\ ${ }^{5}$ Istituto Clinico Humanitas IRCCS, Rozzano, Milan, Italy \\ ${ }^{6}$ Fondazione Studio Diagnostica Nucleare, IRCCS, Naples, Italy \\ ${ }^{7}$ Department of Internal Medicine and Specialist Units, U.O.C. Immunohematology, and Excellence \\ Research Center on Cardiovascular Disease, $1^{\text {st }}$ School of Medicine, \\ Second University of Naples, Naples, Italy \\ ${ }^{8}$ Chair and Division of Geriatrics, University “Federico II”, Naples, Italy \\ ${ }^{9}$ Chair of Cardiac Surgery I, University of Milan, Milan, Italy
}

\begin{abstract}
Background: The use of cardiac resynchronization therapy (CRT) and implantable cardioverter-defibrillator (ICD) for advanced heart failure (HF) is increasing. Renal dysfunction is a common condition in HF which is associated with a worse survival. The study aims at identifying in patients with advanced HF treated with CRT the effect of baseline glomerular filtration rate (GFR), GFR improvement and left ventricular ejection fraction (LVEF) change, after 6-months of CRT implant, on survival.
\end{abstract}

Methods: The study population consisted of 375 advanced HF patients who received a CRT between 1999 and 2009, of these 277 received also an ICD implant. Clinical characteristics (New York Heart Association [NYHA] functional class, ischemic vs. non-ischemic etiology, atrial fibrillation, diabetes, hypertension, LVEF, QRS duration and GFR were recorded. The use of common used drugs was evaluated. Cox proportional hazards analysis was calculated in order to evaluate variables associated to mortality.

Results: During a median follow-up of 43.0 months, 93 (24.8\%) patients died. Patients deceased during the study had at baseline higher NYHA class and lower LVEF and GFR.

\footnotetext{
Address for correspondence: Prof. Claudio Napoli, MD, PhD, MBEth, FACA, Department of Internal Medicine and Specialist Units, U.O.C. Immunohematology, and Excellence Research Center on Cardiovascular Disease, $1^{\text {st }}$ School of Medicine, Second University of Naples, 80138 Naples, Italy, e-mail: cnapoli2@tin.it; claudio.napoli@unina2.it AND

Edoardo Gronda, MD, FESC, Department of Cardiovascular and Metabolic Disease, IRCCS MultiMedica — Via Milanese, 300-20099 Sesto S. Giovanni, Milan, Italy e-mail: edoardo.gronda@multimedica.it
} 
In Cox regression analysis, GFR predicts long-term mortality (hazard ratio [HR] 0.983; 95\% confidence interval [CI] 0.969-0.998; $p=0.023$ ) independently from the effect of others covariates. In addition, a positive GFR improvement 6 months after CRT implant is significantly associated with a lower hazard of mortality (for each $10 \mathrm{~mL} / \mathrm{min}$ of GFR improvement HR 0.86 ; 95\% CI 0.75-0.99; $p=0.038$ ).

Conclusions: GFR is a significant predictor of mortality in advanced HF patients who received CRT. A GFR improvement 6 months after CRT implant is significantly associated with a lower hazard of mortality. (Cardiol J 2015; 22, 4: 459-466)

Key words: cardiac resynchronization therapy, heart failure, outcome, renal function

\section{Introduction}

Controlled trials have demonstrated that cardiac resynchronization therapy (CRT), achieved by biventricular (left ventricular [LV] and right ventricular $[\mathrm{RV}]$ ) pacing improves survival, morbidity, symptoms, quality of life, exercise capacity, cardiac structure and function in heart failure (HF) patients with wide QRS complex [1-8]. However, some patients do not benefit from CRT, particularly those with HF of ischemic etiology [9, 10]. Consequently, extensive research has been conducted to identify predictors of midterm outcomes, such as improvement in New York Heart Association (NYHA) functional class or improvement in LV ejection fraction (LVEF) [6].

Impairment of kidney function is highly prevalent in patients with advanced $\mathrm{HF}$ at the moment of CRT implant and it ranges from $40 \%$ [11] to $71 \%$ [12]. Impaired kidney function has been associated with poor prognosis in patients with HF [13]. Recently, it has been demonstrated that patients who had significant LVEF reverse remodeling after CRT experienced improvements in renal function and survival [11]. However, the independent effect of glomerular filtration rate (GFR) on positive response to CRT treatment and-long term mortality is poorly investigated in $\mathrm{HF}$ patients.

In the present study, we evaluated the effect of kidney function (baseline GFR and GFR improvement after CRT implantation) on mortality in advanced $\mathrm{HF}$ patients who underwent CRT implantation.

\section{Methods}

\section{Patient populations}

Between November 1999 and March 2009, 375 patients with HF who received CRT at the Division of Cardiology, University of Florence, Italy and at the Istituto Clinico Humanitas IRCCS - Rozzano, Milan, Italy were consecutively included in the study. Implantable cardioverter-defibrillator (ICD) were implanted in 277 patients. All the patients had systolic HF and were in NYHA functional class $\geq$ II. Patients in NYHA functional class II received CRT if they were clinically unstable or had recurrent episodes of HF. All patients had a LVEF $\leq 35 \%$ and QRS complex $\geq 120 \mathrm{~ms}$. Patients with primarily significant valve heart disease were excluded. Before CRT, patients were on optimized medical therapy for HF with the maximum tolerated doses of angiotensin-converting enzyme inhibitor (ACEI); angiotensin receptor blockers II (ARB-II), and beta-blockers, and received diuretics as needed to avoid signs and symptoms of fluid retention. The great majority $(99.5 \%)$ of the implants was endocardial and the target pacing site for the LV, the lateral/posterior lateral region, was reached in the majority of the patients. Because indication for ICD therapy changed over time, patients received appropriate device based on currently available evidence and guidelines [6]. The atrio-ventricular pacing interval was adjusted before discharge to optimize hemodynamic parameters during Doppler echocardiography. A complete patients evaluation was done before CRT implantation (baseline) and at the follow-up (6 months) and included the assessment of LVEF and QRS duration, NYHA functional class, etiology (coronary heart disease vs. cardiomyopathy), presence of atrial fibrillation, diabetes, hypertension, and GFR computed using the Modification of Diet in Renal Disease Study equation (MDRD) [14]. GFR improvement was assessed considering the change between basal determination and follow-up.

All 375 patients were followed up and mortality was assessed retrospectively, after CRT implant, until July 2011. A patient with a LVEF increment $\geq 10 \% 6$ months after CRT implantation 
was considered as "Responder". All-cause mortality and hospital admission for decompensate HF were recorded as events. The primary end point was the all cause mortality recorded from family members or from the referring physician.

This study was approved by local Institutional Review Board.

\section{Statistical analysis}

Continuous variables are reported as mean \pm standard deviation. Categorical data are expressed as frequencies and percentages. Two-tailed tests of significance are reported, and $\mathrm{p}$ values lower than 0.05 are considered statistically significant. Univariate comparison of baseline clinical and echo-cardiographic characteristics stratified by allcause mortality was performed by Student's t test or $\chi^{2}$ as appropriate. The Cox proportional hazards $(\mathrm{PH})$ regression model was used to get hazard ratios (HR) and to analyze survival time free from all cause death. Cox PH assumption was evaluated by testing time-varying effect of each covariate. Non linearity in the relation between explanatory and outcome variables has been investigated by the multivariable fractional polynomials (MFP) algorithm [15, 16].

An extension of this algorithm MFP time has been employed to model the time-varying effect when testing the $\mathrm{PH}$ assumption [17]. Model building and variables selection was achieved by subject-matter knowledge and data driven model selection. Age and sex are regarded as "confounders" (as such are forced in the model irrespective of the statistical significance) while all the remaining variables, considered "effect modifiers", are tested before inclusion in the final model. First level interaction effect was planned and tested for the variables (marked with an asterisk in Table 2) by means of the MFP interaction, a further extension of the MFP procedure [18].

The MFP algorithms, employed for variables selection, for non linear continuous variable modeling and for interaction testing, is a systematic approach for multivariate model building based on a closed test procedure which maintains approximately the correct type I error rate [16].

In order to assess the discrimination ability of the final Cox model the $\mathrm{R}^{2}$ measure of the explained variation has been computed [19].

Three $1^{\text {st }}$ level interaction terms, constructed using the variables (marked with an asterisk in Table 2), have been tested in the Cox model. The first and the second, the Responder*GFR and the Responder*LVEF, to assess if being classified as responder exerts a modulation effect on the impact over survival of the GFR, the first, and of the LVEF, the second, (or vice versa). The third, the GFR*LVEF, to asses if varying the levels of one of these variables modify the effect over the survival of the other. None of these interactions reached a statistically significant degree of association.

In the final Cox model that includes the 2 covariates gathered at 6 -month follow-up (namely the GFR improvement and the responder status), 8 patients with missed values of these factors (because of censoring [5 patients] or death [3 patients] before 6 -month follow-up) were excluded from the analysis (listwise missing values exclusion).

All continuous variables, namely the baseline GFR, the GFR improvement and the LVEF, were tested for linearity of the effect by means of the fractional polynomial algorithm $[15,16]$ none showed a significant non linear association.

The final Cox model, built in order to evaluate the effect on mortality, includes, in addition to the a priori incorporated age and sex as confounder variables, all the variables tested as effect modifier showing a statistically significant level.

All the variables included in the final model were tested for the $\mathrm{PH}$ assumption, none violated it. In order to have a comprehensive view of the relative weight of each factor on the survival curve, the HR of the significant continuous variables included in the final Cox models were related to clinically meaningful variations, $10 \mathrm{~mL} / \mathrm{min}$ for basal GFR and GFR improvement, 5\% for LVEF.

Statistical analysis was performed with the STATA 11.2 software (Stata Corp LP).

\section{Results}

Mean age of the 375 patients with advanced $\mathrm{HF}$ was $66.6 \pm 10.1$ years (range $21-86$ ). All patients were observed for a median of 43.0 months (min: 2 , max: 135), 93 (24.8\%) patients died. Mortality rate at 1 and 5 years was $3.1 \%$ and $29.4 \%$, respectively. All patients had depressed LVEF $(28.0 \pm 5.4)$, symptomatic advanced HF (NYHA I: 0\%, NYHA II: 18.9\%, NYHA III: $65.3 \%$, NYHA IV: $15.7 \%$ ), and a wide QRS complex $(154.7 \pm 30.5 \mathrm{~ms})$. Atrial fibrillation was present in $15.7 \%$, while ischemic HF was present in 183 (48.8\%) patients. ICD was implanted in 277 patients. Mean GFR was $59.9 \pm$ \pm 23.5 and 197 (52.7\%) had chronic kidney disease (CKD) (defined as a GFR of $\leq 60 \mathrm{~mL} / \mathrm{min} / 1.73 \mathrm{~m}^{2}$ ) at the time of device implantation. Prevalence of patient with a LVEF increment $\geq 10 \% 6$ months after CRT implantation ("Responder") was $36.3 \%$ 
Table 1. Baseline characteristics of 375 patients enrolled in the study.

\begin{tabular}{|c|c|c|c|c|}
\hline Clinical variables & All $(n=375)$ & Survived (n = 295) & $\operatorname{Died}(n=80)$ & $\mathbf{P}$ \\
\hline Age [years] & $66.6 \pm 10.1$ & $66.3 \pm 10.3$ & $67.8 \pm 9.3$ & 0.249 \\
\hline Men & $303(80.8 \%)$ & $239(81.0 \%)$ & $64(80.0 \%)$ & 0.838 \\
\hline QRS duration [ms] & $154.7 \pm 30.5$ & $154.4 \pm 27.5$ & $156.1 \pm 40.7$ & 0.687 \\
\hline Atrial fibrillation & $59(15.7 \%)$ & $40(13.6 \%)$ & $19(23.8 \%)$ & 0.026 \\
\hline NYHA functional class & $3.0 \pm 0.6$ & $2.9 \pm 0.6$ & $3.2 \pm 0.6$ & 0.000 \\
\hline \multicolumn{5}{|c|}{ Etiology (CHD vs. cardiomyopathy) } \\
\hline $\mathrm{CHD}$ & $183(48.8 \%)$ & $150(50.8 \%)$ & $42(52.8 \%)$ & 0.793 \\
\hline \multicolumn{5}{|l|}{ Medical therapy: } \\
\hline ICD & $277(73.9 \%)$ & $228(77.3 \%)$ & $49(61.3 \%)$ & 0.004 \\
\hline Beta-blockers & $284(75.7 \%)$ & $237(80.3 \%)$ & $47(58.8 \%)$ & 0.000 \\
\hline ACEI & $260(69.3 \%)$ & $209(70.8 \%)$ & $51(63.8 \%)$ & 0.222 \\
\hline ARB-II & $86(22.9 \%)$ & $68(23.1 \%)$ & $18(22.5 \%)$ & 0.917 \\
\hline Diuretics & $344(91.7 \%)$ & $269(91.2 \%)$ & $75(93.8 \%)$ & 0.460 \\
\hline Digoxin & $138(36.8 \%)$ & $90(32.5 \%)$ & $42(53.2 \%)$ & 0.001 \\
\hline Amiodarone & $87(23.2 \%)$ & $64(21.7 \%)$ & $23(29.1 \%)$ & 0.166 \\
\hline LVEF [\%] & $28.0 \pm 5.40$ & $28.5 \pm 5.1$ & $26.1 \pm 6.2$ & 0.000 \\
\hline Basal GFR MDRD & $59.9 \pm 23.5$ & $61.6 \pm 24.2$ & $53.4 \pm 19.4$ & 0.005 \\
\hline GFR improvement & $0.92 \pm 17.8$ & $1.68 \pm 17.4$ & $-1.37 \pm 18.9$ & 0.150 \\
\hline Diabetes & $106(28.3 \%)$ & $81(27.5 \%)$ & $25(31.3 \%)$ & 0.504 \\
\hline Hypertension & $185(49.3 \%)$ & $154(52.2 \%)$ & $31(38.8)$ & 0.033 \\
\hline Responder ( $\uparrow$ LVEF 10\%) & $136(36.3 \%)$ & $103(37.2 \%)$ & $31(33.3)$ & 0.497 \\
\hline
\end{tabular}

Mean \pm standard deviation if continuous variable, number of elements and percent of the overall if counting variable; NYHA - New York Heart Association; CHD — coronary heart disease; ICD — implantable cardioverter-defibrillator; ACEI — angiotensin-converting enzyme inhibitor; ARB-II - angiotensin receptor blockers II; LVEF — left ventricular ejection fraction; GFR — glomerular filtration rate; Basal GFR MDRD - GFR from Modification of Diet in Renal Disease (MDRD) Study equation; GFR improvement - change between basal determination and follow-up; Responder - a patient with a LVEF increment $\geq 10 \% 6$ months after CRT implantation

(Table 1). Diuretics were used in $344(91.7 \%)$ patients, beta-blockers in $284(75.7 \%)$, ACEI in 260 (69.3\%) and ARB-II in 86 patients (22.9\%).

The final Cox PH regression model (Table 2) shows that having a baseline GFR $10 \mathrm{~mL} / \mathrm{min}$ greater is significantly associated with a $12 \%$ smaller hazard of all cause cumulative mortality (for each $10 \mathrm{~mL} / \mathrm{min}$ increase HR $0.88 ; 95 \% \mathrm{CI}$ $0.78-0.98 ; p=0.003)$. In addition, a positive GFR improvement 6 months after CRT implant is significantly associated with a lower mortality risk (for each $10 \mathrm{~mL} / \mathrm{min}$ of GFR improvement HR 0.86 ; 95\% CI 0.75-0.99; $\mathrm{p}=0.038$ ). Moreover, patients experiencing a 6 months improvement of GFR show a positive effect on survival independently from the baseline values. Higher LVEF (for each $5 \%$ LVEF increase HR 0.65; 95\% CI 0.53-0.79; $\mathrm{p}<0.001$ ) and use of beta-blockers (HR $0.57 ; 95 \%$ CI $0.37-0.88 ; \mathrm{p}=0.013$ ) are associated with lower hazard. Interestingly, patients presenting with the diagnosis of hypertension showed a lower hazard (HR 0.55; 95\% CI 0.35-0.85; $\mathrm{p}=0.015$ ). Being classified as responder to CRT implant resulted in a significant $57 \%$ lower hazard (HR $0.57,95 \%$ CI 0.35-0.93).

The $\mathrm{R}^{2}$ computed for the final model (a measure - on the log relative hazard scale - of the variation explained by the covariates included in the model) is 0.28 , i.e. almost one third of the variance is explained by the variables included in the model. Figures 1 and 2 show survival curves relative to, respectively, three baseline GFR levels and three different GFR improvements 6 months after CRT implant, namely one with no changes and, of the remaining two, one improving and one declining both by an amount equal to one standard deviation $(18 \mathrm{~mL} / \mathrm{min})$.

\section{Discussion}

The study supports the evidence that renal dysfunction at the time of CRT implant is associated with a poorer overall survival. The magnitude of the HR involved $12 \%$ smaller hazard every 
Table 2. Cox multivariate regression on mortality.

\begin{tabular}{lcc}
\hline Variables & HR (95\% CI) & P \\
\hline Age & $1.01(0.99-1.04)$ & - \\
Male sex & $0.96(0.56-1.66)$ & 0.884 \\
Etiology (CAD vs. cardiomyopathy) & $1.04(0.65-1.64)$ & 0.195 \\
NYHA functional class & $1.29(0.88-1.89)$ & 0.380 \\
Atrial fibrillation & $1.25(0.76-2.09)$ & 0.397 \\
ICD & $0.81(0.49-1.32)$ & 0.013 \\
Beta-blockers & $0.57(0.37-0.88)$ & 0.237 \\
ACEI & $0.76(0.49-1.18)$ & 0.174 \\
ARB-II & $0.64(0.34-1.22)$ & 0.590 \\
Diuretics & $1.27(0.54-2.98)$ & 0.966 \\
Digoxin & $1.01(0.64-1.58)$ & 0.386 \\
Amiodarone & $1.24(0.76-2.02)$ & $<0.001$ \\
LVEF (HR relative to $5 \%$ change) & $0.65(0.54-0.79)$ & 0.003 \\
Basal GFR (HR relative to $10 \mathrm{~mL} / \mathrm{min})^{*}$ & $0.88(0.78-0.98)$ & 0.038 \\
GFR improvement (HR relative to $10 \mathrm{~mL} / \mathrm{min})$ & $0.86(0.75-0.99)$ & 0.415 \\
Diabetes mellitus & $1.22(0.75-1.96)$ & 0.008 \\
Hypertension & $0.54(0.35-0.85)$ & 0.011 \\
Responder ( $\uparrow$ LVEF $\geq 10 \%)^{*}$ & $0.57(0.35-0.93)$ & \\
\hline
\end{tabular}

*The asterisk mark variables involved in $1^{\text {st }}$ level interaction assessment; $\mathrm{HR}$ - hazard ratio; $\mathrm{Cl}$ - confidence interval; CHD - coronary heart disease; ICD - implantable cardioverter-defibrillator; ACEI — angiotensin-converting enzyme inhibitor; ARB-II - angiotensin receptor blockers II; LVEF - left ventricular ejection fraction; GFR - glomerular filtration rate; Basal GFR — GFR from Modification of Diet in Renal Disease (MDRD) Study equation; GFR improvement (HR relative to $10 \mathrm{~mL} / \mathrm{min}$ change) — change between basal determination and follow-up; Responder - a patient with a LVEF increment $\geq 10 \% 6$ month after CRT implantation

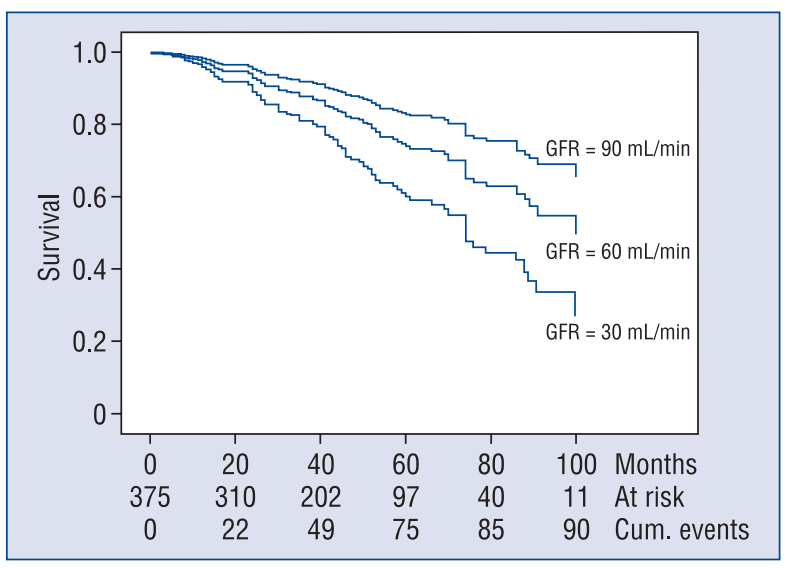

Figure 1. Cumulative survival to all-cause death for three different levels of glomerular filtration rate (GFR) adjusted for all the remaining variables of the final Cox model.

$10 \mathrm{~mL} / \mathrm{min}$ of GFR increase, denotes a clinically moderate effect exerted by impaired renal function on mortality in patients with advanced $\mathrm{HF}$ after CRT implant. This consideration becomes relevant in view of the high prevalence of kidney dysfunction in $\mathrm{HF}$ patients, $52.7 \%$ in our study. Moreover,

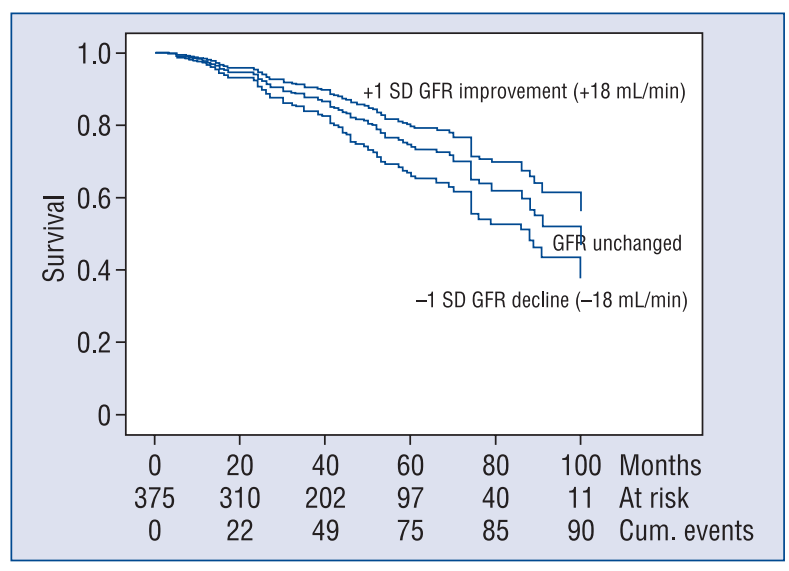

Figure 2. Cumulative survival to all-cause death for three different levels ( $0 \pm 1$ standard deviation [SD]) of glomerular filtration rate (GFR) improvement adjusted for all the remaining variables of the final Cox model.

patients experiencing a 6 months improvement of GFR show a positive effect on survival that, indeed, is independent from the baseline values, supporting the proposition that therapies oriented to ameliorate kidney function are potentially beneficial to the overall survival. Moreover, baseline GFR is 
a predictor of positive response to the CRT therapy (i.e. heart pump function improvement), resulting in a patient with a relevant (10\%) increase of the LVEF 6 months after CRT implant. All these evidences make GFR an important parameter in the evaluation of CRT implant, suggesting that CRT implant should be performed before evident kidney dysfunction if we positively modify prognosis.

\section{Heart failure, renal function and CRT}

Our study shows that a large part of patients with advanced $\mathrm{HF}(52.7 \%)$ had CKD at the time of device implantation, confirming data from previous studies, where CKD was highly prevalent among $\mathrm{HF}$ patients. It has been estimated that as many as $25 \%$ to $50 \%$ of patients with $\mathrm{HF}$ have impaired renal function (creatinine clearance $<60-75 \mathrm{~mL} /$ $/ \mathrm{min} / 1.73 \mathrm{~m}^{2}$ ) [20-22]. Recently, a systematic review has examined the effects of CRT in CKD patients (eGFR $<60 \mathrm{~mL} / \mathrm{min} / 1.73 \mathrm{~m}^{2}$ ) with HF. Eighteen studies (14 observational studies and 4 randomized clinical trials) were considered. The analysis demonstrated a modest improvement in estimated GFR with CRT among CKD patients (mean difference $2.30 \mathrm{~mL} / \mathrm{min} / 1.73 \mathrm{~m}^{2}$ ). Similarly, there was a significant improvement in LVEF with CRT in CKD patients (mean difference $6.24 \%$ ). Subgroup analysis of 3 randomized clinical trials reported lower rates of death or hospitalization for $\mathrm{HF}$ with CRT in the CKD population [23].

\section{Heart failure, renal function, CRT and mortality}

GFR improvement was associated with improved survival.

Moreover, our findings are consistent with previous observations that worsening renal function confers worse outcomes in HF. Data derived from the SOLVD (Studies of LV Dysfunction) trial revealed that in HF patients with a LVEF $<35 \%$, a total of $32 \%$ of patients had a GFR $<60 \mathrm{~mL} / \mathrm{min} / 1.73 \mathrm{~m}^{2}$, moreover, a $10 \mathrm{~mL} / \mathrm{min} / 1.73 \mathrm{~m}^{2}$ lower GFR was associated with a 1.064 (95\% CI 1.033-1.096) higher risk of mortality in these patients [24]. The currently observed HR for GFR (for each $10 \mathrm{~mL} / \mathrm{min}$ increase HR 0.88; 95\% CI 0.78-0.98; $\mathrm{p}=0.003$ ) is fairly higher than that reported by SOLVD, but may be attributed to the fact that in the SOLVD trial, only $12 \%$ of patients were in NYHA functional class III or IV, vs. $81 \%$ in our study. Similar observations as in SOLVD were noted in another large trial, CHARM (Candesartan in Heart Failure: Assessment of Reduction in Mortality and Morbidity) [25], where around $35 \%$ of 2,680 patients had a GFR
$<60 \mathrm{~mL} / \mathrm{min} / 1.73 \mathrm{~m}^{2}$, and this was associated with increased mortality during long-term follow-up (HR 1.50; 95\% CI 1.15-2.00) [25].

Lin et al. [12] demonstrate in 482 CRT device implantations, $71 \%$ with CKD (defined as a GFR of $\leq 60 \mathrm{~mL} / \mathrm{min} / 1.73 \mathrm{~m}^{2}$ ), that CKD at the time of CRT implantation was associated with worse survival after 3 years of follow-up ( 57 vs. $72 \%$; $p<0.01$ ). In our study, we observed a lower mortality $(29.4 \%$ after 5 years of follow-up) and this could be in part due to the lower rate of CKD prevalence in our sample (52.7 vs. $71 \%$ ) and the younger age (66 vs. 71$)$.

\section{Renal function improvement after CRT and mortality}

Estimated GFR improved modestly in patients with baseline CKD (mean change $0.95 \pm 8.71 \mathrm{~mL} /$ $/ \mathrm{min} / 1.73 \mathrm{~m}^{2}$ ) and decreased modestly in patients without baseline CKD (mean change GFR -1.99 $\left.\pm 11.23 \mathrm{~mL} / \mathrm{min} / 1.73 \mathrm{~m}^{2}, \mathrm{p}=0.03\right)$. A similar trend is observed in our study where estimated GFR improved $0.92 \pm 17.8 \mathrm{~mL} / \mathrm{min} / 1.73 \mathrm{~m}^{2}$, after 6 months of CRT implant. In patients with baseline CKD, there was an improvement (mean change $4.24 \pm$ $\pm 14.2 \mathrm{~mL} / \mathrm{min} / 1.73 \mathrm{~m}^{2}$ ), while in patients without baseline CKD there was a decrease (mean change GFR $\left.-3.01 \pm 20.9 \mathrm{~mL} / \mathrm{min} / 1.73 \mathrm{~m}^{2}, \mathrm{p}=0.00\right)$ (data not shown).

Adelstein et al. [26] found a similar result to our study among CRT-D recipients, overall survival improved incrementally with higher baseline GFR (for each $10 \mathrm{~mL} / \mathrm{min} / 1.73 \mathrm{~m}^{2}$ increase, corrected HR 1.21; 95\% CI 1.13-1.30; p < 0.0001). Moreover, the survival benefit was associated with improved renal and cardiac function. CRT recipients with GFR $\geq 60 \mathrm{~mL} / \mathrm{min} / 1.73 \mathrm{~m}^{2}$ derived significant echocardiographic benefit but experienced a GFR decline, whereas those with GFR $<30 \mathrm{~mL} / \mathrm{min} / 1.73 \mathrm{~m}^{2}$ had no echocardiographic benefit but an improvement in GFR. Similarly, we found that a positive GFR improvement, 6 months after CRT implant, is significantly associated with a lower hazard (for each $10 \mathrm{~mL} / \mathrm{min} / 1.73 \mathrm{~m}^{2}$ of GFR improvement HR 0.86; 95\% CI 0.75-0.99; $\mathrm{p}=0.038$ ). A similar result is also found by Hosoda et al. [20] who demonstrated in 67 consecutive patients who underwent CRT that advanced renal insufficiency was an independent predictor of cardiac mortality combined with HF hospitalization (odds ratio 3.01, $\mathrm{p}=0.008$ ). Subgroup analysis in the baseline advanced renal insufficiency group revealed that patients with preserved renal function by CRT $(<10 \%$ reduction in estimated GFR) 
had a higher rate of decrease of LV end-systolic diameter $(-14.0 \%$ vs. $-0.8 \%, p=0.023)$ and lower cardiac mortality combined with HF hospitalization (log-rank: $p=0.029$ ) compared with patients with deterioration of renal function $(\geq 10 \%$ reduction in estimated GFR). These results are in line with our observation, we demonstrated that different GFR changes 6 months after CRT implant are an independent predictor of mortality (HR $0.86 ; 95 \%$ CI 0.75-0.99; $\mathrm{p}=0.038$ ).

An unresolved question is if renal impairment associated with HF could be improved by CRT and this effect could improve survival. Our study demonstrated that the proportion of patients in whom estimated GFR improved after CRT implant experienced lower mortality, supporting the proposition that therapies oriented to ameliorate kidney function are potentially beneficial to the overall survival, independently from cardiac function. Kidney function is strongly influenced by renal perfusion improved by CRT which could induce systemic hemodynamic benefits. This hemodynamic benefit is most likely exerted by improvement in LV systolic function and/or reduction in mitral regurgitation. Fung et al. [21] reported that, after 3 months of CRT, patients with reverse remodeling (defined as at least $10 \%$ reduction in LV end-systolic volume), had a mean increase of GFR from an average of 51.7 to $54.2 \mathrm{~mL} / \mathrm{min} / 1.73 \mathrm{~m}^{2}$, whereas patients without reverse remodeling had a decrease from a mean $61.9-48.8 \mathrm{~mL} / \mathrm{min} / 1.73 \mathrm{~m}^{2}$. The major limitation of this study was the lack of a control group. Van Bommel et al. [11] in a subset of 133 patients, with 6 -month blood sample data, observed a significant deterioration in renal function (GFR change, $-4.7 \mathrm{~mL} / \mathrm{min} / 1.73 \mathrm{~m}^{2}$ ) among patients who did not respond to CRT with LV reverse remodeling, whereas GFR did not change significantly during the 6 months of follow-up among CRT responders. Recently, Eisen et al. [22] demonstrated on 730 ICD and 453 CRT-D patients that mean estimated GFR after 1-year follow-up decreased by $8.0 \pm 4.3 \mathrm{~mL} /$ $/ \mathrm{min} / 1.73 \mathrm{~m}^{2}$ in ICD patients $(\mathrm{p}=0.06)$ and by $1.8 \pm 1.3 \mathrm{~mL} / \mathrm{min} / 1.73 \mathrm{~m}^{2}$ in patients with CRT-D $(p=0.2)$. The GFR changes differed significantly between CRT responders and non-responders suggesting that this clinical parameter could help to determine which patient will respond to CRT, and which patient might not respond indicating a pathogenic interconnection between heart and kidney [11, 27-29].

In our study, we demonstrate that a positive GFR improvement 6 months after CRT implant is significantly associated with a lower hazard of mortality (for each $10 \mathrm{~mL} / \mathrm{min}$ of GFR improvement HR 0.86; 95\% CI 0.75-0.99; $\mathrm{p}=0.038$ ).

\section{Limitations of the study}

Some limitations of the present study have to be addressed. First, the present report is based on a retrospective approach. Therefore, the observation we made cannot prove causality per se. Second, the choice to implant CRT-D vs. CRT-P may affect the survival and may constitute a bias difficult to sort out even with control in the multivariate model. Third, the exclusion of 8 patients censored before or not surviving 6 months of follow-up may represent a "survival bias", however, the small amount of patients involved, and the lack of a specific distinctive tract in these patients (LVEF and basal GFR in the 3 deceased and in the 5 censored are LVEF 26.6 \pm 6.0, basal GFR $57.7 \pm 29.9$ and LVEF $28.4 \pm 5.1$, basal GFR $60.6 \pm 20.9$, respectively) suggest that a negligible influence on the overall results that we observed is expected.

\section{Conclusions}

In our study population, we demonstrate that GFR predicts long-term mortality in patients with advanced HF treated with CRT. The baseline GFR differed significantly between CRT responders and non-responders suggesting that this clinical parameter could help to determine which patient will respond to CRT, and in turn could affect mortality. A GFR improvement 6 months after CRT implant is significantly associated with a lower hazard of mortality. Whether early placement of CRT will also determine delayed onset of CKD, and a possible mortality benefit, deserves further study.

\section{Conflict of interest: None declared}

\section{References}

1. Abraham WT, Fisher WG, Smith AL et al. Multicenter InSync Randomized Clinical Evaluation. Cardiac resynchronization in chronic heart failure. N Engl J Med, 2002; 346: 1845-1853.

2. Young JB, Abraham WT, Smith AL et al. Multicenter InSync ICD Randomized Clinical Evaluation (MIRACLE ICD) Trial Investigators. Combined cardiac resynchronization and implantable cardioversion defibrillation in advanced chronic heart failure: The MIRACLE ICD Trial. JAMA, 2003; 289: 2685-2694.

3. Sutton MG, Plappert T, Hilpisch KE, Abraham WT, Hayes DL, Chinchoy E. Sustained reverse left ventricular structural remodelling with cardiac resynchronization at one year is a function of etiology: Quantitative Doppler echocardiographic evidence from the MulticenterInSync Randomized Clinical Evaluation (MIRACLE). Circulation, 2006; 113: 266-272. 
4. Higgins SL, Hummel JD, Niazi IK et al. Cardiac resynchronization therapy for the treatment of heart failure in patients with intraventricular conduction delay and malignant ventricular tachyarrhythmias. J Am Coll Cardiol, 2003; 42: 1454-1459.

5. Auricchio A, Stellbrink C, Butter C et al. Pacing Therapies in Congestive Heart Failure (PATH-CHF) Study Group. Long-term clinical effect of hemodynamically optimized cardiac resynchronization therapy in patients with heart failure and ventricular conduction delay. J Am Coll Cardiol, 2002; 39: 2026-2033.

6. Stevenson WG, Hernandez AF, Carson PE et al. Heart Failure Society of America Guideline Committee. Indications for cardiac resynchronization therapy: 2011 update from the Heart Failure Society of America Guideline Committee. J Card Fail, 2012; 18: 94-106.

7. Bristow MR, Saxon LA, Boehmer J et al. Cardiac-resynchronization therapy with or without an implantable defibrillator in advanced chronic heart failure. N Engl J Med, 2004; 350: 2140-2150.

8. Cleland JG, Daubert JC, Erdmann E et al. Cardiac Resynchronization-Heart Failure (CARE-HF)Study Investigators. The effect of cardiac resynchronization on morbidity and mortality in heart failure. N Engl J Med, 2005; 352: 1539-1549.

9. Abraham WT, Hayes DL. Cardiac resynchronization therapy for heart failure. Circulation, 2003; 108: 2596-2603.

10. Leclercq C, Kass DA. Retiming the failing heart: principles and current clinical status of cardiac resynchronization. J Am Coll Cardiol, 2002; 39: 194-201.

11. Van Bommel RJ, Mollema SA, Borleffs CJ et al. Impaired renal function is associated with echocardiographic nonresponse and poor prognosis after cardiac resynchronization therapy. J Am Coll Cardiol, 2011; 57: 549-555.

12. Lin G, Gersh BJ, Greene EL, Redfield MM, Hayes DL, Brady PA. Renal function and mortality following cardiac resynchronization therapy. Eur Heart J, 2011; 32: 184-190.

13. Smith GL, Lichtman JH, Bracken MB et al. Renal impairment and outcomes in heart failure: Systematic review and meta-analysis. J Am Coll Cardiol, 2006; 47: 1987-1996.

14. Miller WG. Estimating glomerular filtration rate. Clin Chem Lab Med, 2009; 47: 1017-1019.

15. Royston P, Ambler G, Sauerbrei W. The use of fractional polynomials to model continuous risk variables in epidemiology. Int J Epidemiol, 1999; 28: 964-974.

16. Sauerbrei W, Meier-Hirmer C, Benner A. Multivariable regression model building by using fractional polynomials: description of SAS, STATA and R programs. Comput Stat Data An, 2006; 50: 3464-3485.

17. Sauerbrei W, Royston P, Look M. A new proposal for multivariable modelling of time-varying effects in survival data based on fractional polynomial time-transformation. Biom J, 2007; 49: 453-473.

18. Royston P, Sauerbrei W. A pragmatic approach to regression analysis based on fractional polynomials for modeling continuous variables. In: Royston P, Sauerbrei W eds. Multivariate model building. Wiley, Chichester, U: 2008: 89-96.

19. Royston P. Explained variation for survival models. Stata J, 2006; 6: 83-96.

20. Hosoda J, Ishikawa T, Matsushita $\mathrm{K}$ et al. Impact of renal insufficiency on long-term clinical outcome in patients with heart failure treated by cardiac resynchronization therapy. J Cardiol, 2012; 60: 301-305.

21. Fung JW, Szeto CC, Chan JY et al. Prognostic value of renal function in patients with cardiac resynchronization therapy. Int J Cardiol, 2007; 122: 10-16.

22. Eisen A, Suleiman M, Strasberg B et al. Renal dysfunction and clinical outcomes of patients undergoing ICD and CRTD implantation: data from the Israeli ICD registry. J Cardiovasc Electrophysiol, 2014; 25: 990-997.

23. Garg N, Thomas G, Jackson G et al. Cardiac resynchronization therapy in CKD: A systematic review. Clin J Am Soc Nephrol, 2013; 8: 1293-1303.

24. Al-Ahmad A, Rand WM, Manjunath G et al. Reduced kidney function and anemia as risk factors for mortality in patients with left ventricular dysfunction. J Am Coll Cardiol, 200; 38: 955-962.

25. Hillege HL, Nitsch D, Pfeffer MA et al. Renal function as a predictor of outcome in a broad spectrum of patients with heart failure. Circulation, 2006; 113: 671-678.

26. Adelstein EC, Shalaby A, Saba S. Response to cardiac resynchronization therapy in patients with heart failure and renal insufficiency. Pacing Clin Electrophysiol, 2010; 33: 850-859.

27. Napoli C, Casamassimi A, Crudele V, Infante T, Abbondanza C. Kidney and heart interactions during cardiorenal syndrome: a molecular and clinical pathogenic framework. Future Cardiol, 2011; 7: 485-497.

28. Abete P, Testa G, Della-Morte D et al. Treatment for chronic heart failure in the elderly: current practice and problems. Heart Fail Rev, 2013; 18: 529-551.

29. Testa G, Della-Morte D, Cacciatore F et al. Precipitating factors in younger and older adults with decompensated chronic heart failure: Are they different? J Am Geriatr Soc, 2013; 61: 1827-1828. 